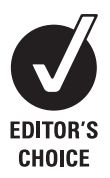

${ }^{1}$ Department of Surgery, North Tyneside General Hospital, North Shields, UK; ${ }^{2}$ Human Nutrition Research Centre, Institute for Ageing and Health, Newcastle University, Newcastle Upon Tyne, UK; ${ }^{3}$ Institute of Human Genetics, Centre for Life,

Newcastle University,

Newcastle Upon Tyne, UK

Correspondence to:

Mr S S Dronamraju, Department of General Surgery, North Tyneside General Hospital, Rake Lane, North Shields NE29 8NH, UK; mailshridhar@yahoo.com

Revised 12 September 2008 Accepted 14 October 2008 Published Online First

31 October 2008

\title{
Cell kinetics and gene expression changes in colorectal cancer patients given resistant starch: a randomised controlled trial
}

\author{
S S Dronamraju, ${ }^{1}$ J M Coxhead, ${ }^{2}$ S B Kelly, ${ }^{1}$ J Burn, ${ }^{3}$ J C Mathers ${ }^{2}$
}

\begin{abstract}
Objective: This study investigated the effects of oral supplementation of resistant starch (RS) on tumour cell and colonic mucosal cell kinetics and on gene expression in patients with colorectal cancer (CRC), and its potential role in colon cancer prevention.
\end{abstract}

Methods: 65 patients with CRC were randomised to treatment with RS or ordinary starch (OS) and were given starch treatment for up to 4 weeks. Pretreatment and post-treatment biopsies were obtained from the tumour and colonic mucosa, and the effects of the starch treatment on cell proliferation and expression of the cell cycle regulatory genes CDK4 (cyclin-dependent kinase 4) and GADD45A (growth arrest and DNA damage-inducible, alpha) were investigated.

Results: The proportion of mitotic cells in the top half of the colonic crypt was significantly lower following RS treatment (3.1 (1.5), mean (SEM)) as compared with OS treatment (13.7 (3.2)) ( $p=0.028)$. However, there was no effect of RS treatment on crypt dimensions and tumour cell proliferation index. There was significant upregulation in expression of CDK4 $(\mathrm{p}<0.01)$ and downregulation in expression of GADD45A $(p<0.001)$ in the tumour tissue when compared with macroscopically normal mucosa. Following RS treatment, CDK4 expression in tumours $(0.88(0.15))$ was twofold higher than that in the OS group $(0.37(0.16))(p=0.02)$. The expression of GADD45A, which was downregulated in the presence of cancer, was significantly upregulated $(p=0.048)$ following RS treatment $(1.41(0.26))$ as compared with OS treatment $(0.56(0.3))$. However, there were no significant differences in the expression of these genes in the normal mucosa following starch treatment.

Conclusions: Cell proliferation in the upper part of colonic crypts is a premalignant marker and its reduction by RS supplementation is consistent with an antineoplastic action of this food component. Differential expression of the key cell cycle regulatory genes may contribute to the molecular mechanisms underlying these antineoplastic effects of RS.

Trial registration number: ISRCTN93586244.

Resistant starch (RS) is defined as the fraction of dietary starch which resists digestion in the small intestine of healthy individuals. ${ }^{1}$ This undigested starch forms a major substrate for microbial fermentation in the large bowel, leading to the production of short chain fatty acids (SCFAs) including acetate, propionate and butyrate. ${ }^{23} \mathrm{RS}$ has a number of physiological effects in the colon, most of which are thought to be due to the SCFAs, particularly butyrate. There is compelling evidence that butyrate causes a dose-dependent suppression of proliferation of colon cancer cell lines, ${ }^{4-8}$ and RS has antineoplastic effects against colon cancer in animal models. ${ }^{9-11}$ Recent studies in rat have shown that feeding RS reduced the incidence and multiplicity of azomethane-induced colon cancers and reduced the tumour-enhancing effects of indigestible protein and red meat. ${ }^{12-14}$ These protective effects of RS were thought to be secondary to fermentation of RS to butyrate in the colon.

A limited number of studies have investigated the antineoplastic effects of RS in vivo in humans. The results of these studies, which included mostly healthy human volunteers, have been inconclusive. Van Munster et al ${ }^{3}$ reported a significant reduction in colonic mucosal cell proliferation. Although most of the other human studies reported an increase in the concentrations of SCFAs and reduction in the concentration of bile salts in the colonic lumen, there were no significant effects on mucosal cell proliferation. ${ }^{15-17}$

The mechanism(s) responsible for the antineoplastic effects of butyrate is not known with certainty, but butyrate influences several processes important in tumourigenesis, including cell proliferation, apoptosis, angiogenesis, immunosurveillance and inflammation. These effects of butyrate have been attributed to changes in gene expression ${ }^{17-20}$ possibly as a consequence of epigenetic processes including hyperacetylation of histones $\mathrm{H} 3, \mathrm{H} 4, \mathrm{H} 2 \mathrm{~A}$ and $\mathrm{H} 2 \mathrm{~B}$. Our recent work investigating the possible mechanisms for chemoprevention of DNA mismatch repair-deficient colorectal cancer (CRC) highlighted the enhanced antineoplastic effects and differential gene expression in such tumour cells following butyrate treatment. $^{21}$

This study was undertaken to investigate the effects on cell kinetics and gene expression in both colonic tumours and the adjacent macroscopically normal appearing flat mucosa following oral supplementation of RS in patients with CRC. Gene expression changes were investigated in two cancer-related genes, cyclin-dependent kinase 4 (CDK4) and growth arrest and DNA damageinducible, alpha (GADD45A). These two genes are differentially expressed following butyrate treatment in in vitro studies on colon cancer cells. ${ }^{20-22}$

\section{PATIENTS AND METHODS}

\section{Patient enrolment}

Adult patients undergoing colonoscopy or a flexible sigmoidoscopy with sympoms highly suspicious of CRC and patients with a histologyproven CRC amenable for surgical treatment were 
considered for the study. Enrolment of patient volunteers was undertaken at two District General Hospitals in North East England from July 2005 to May 2006. Patients who had a previous subtotal colectomy or ileostomy, pregnant women, patients receiving long course preoperative chemo-radiotherapy for rectal cancers, patients with symptoms suggestive of bowel obstruction and patients needing emergency surgery for their cancer were excluded from the study.

\section{Study protocol}

This was a randomised double-blind placebo-controlled intervention trial using two different study protocols-that is, Study 1 and Study 2. In Study 1, patients were consented and recruited at the time of colonoscopy before the diagnosis of CRC. Pretreatment biopsies were obtained from the normal mucosa and from the suspected cancer at the time of colonoscopy, and only patients with histology-proven CRC amenable for surgical resection were retained in the study. Patients were then randomised (www.randomizer.org) into two groups: (1) treatment (resistant starch (RS)) group and (2) placebo (ordinary starch $(\mathrm{OS})$ ) group. Volunteers were allotted to treatment and placebo groups in a ratio of $2: 1$, respectively, and were given starch treatments as explained below. In Study 2, volunteers were consented and recruited into the study after the histological diagnosis of CRC, and no preoperative biopsies were obtained in this group of volunteers. Volunteers were randomised into treatment (RS) and placebo (OS) groups as described above. Post-treatment biopsies from the tumour and from the macroscopically normal mucosa at least $5 \mathrm{~cm}$ from the tumour margin were obtained from the resected colectomy specimens. Biopsies were placed in three sets of cryovials containing RNAlater (Ambion, Applied Biosystems, Warrington, UK), Carnoy's solution (ethanol:acetic acid 75:25) (Sigma Aldrich, York, UK) and formalin (Sigma Aldrich), respectively. Tissue in RNAlater was kept at $4^{\circ} \mathrm{C}$ for $4-6 \mathrm{~h}$, snap-frozen in liquid nitrogen and stored at $-80^{\circ} \mathrm{C}$ until required for studying gene expression. Tissue in Carnoy's solution was transferred to $70 \%$ ethanol within $12-24 \mathrm{~h}$ of collection and stored at $4^{\circ} \mathrm{C}$ until used for measurements of crypt cell kinetics. Formalin-fixed paraffinembedded tissues were used for immunohistochemical studies.

\section{Study intervention: starch treatment}

The starches used in this study were food-grade starches provided by the National Starch and Chemical Company, New Jersey, USA (www.nationalstarch.com). The test starch (RS) was a 1:1 blend of Novelose 240 and Novelose 330. Novelose 240 contains approximately 70\% amylose and 30\% amylopectin, and is a rich source of granular resistant starch. Novelose 330 is a good source of non-granular resistant starch. The placebo starch (OS) was Amioca waxy starch which contains only amylopectin. Because of its branched and open structure, amylopectin is rapidly and completely digested in the small bowel. In contrast, amylose has a linear molecular structure which forms helical structures and, without gelatinisation, is resistant to pancreatic amylase. ${ }^{23}$ Study participants were provided with presealed boxes containing the appropriate starch sachets (RS or OS) and were asked to take $30 \mathrm{~g}$ of starch (two sachets) per day until the day before the planned surgery. Patients were provided with 4 weeks supply of starch and were contacted weekly to ensure compliance with treatment. Subjects who took the starch treatment for $<2$ weeks were excluded from the final analysis.
Table 1 Primer sequences for the 18S, CDK4 and GADD45 genes

\begin{tabular}{lll}
\hline Target gene & $\begin{array}{l}\text { Gene accession } \\
\text { no. }\end{array}$ & Primer sequence $\left(\mathbf{5}^{\prime}\right.$ to $\left.\mathbf{3}^{\prime}\right)$ \\
\hline $18 S$ & NM_011552 & $\begin{array}{l}\text { Forward-AGGAATTGACGGAAGGGCACCAC } \\
\text { Reverse-GTGCAGCCCCGACATCTAAGG }\end{array}$ \\
CDK4 & NM_000075 & $\begin{array}{l}\text { Forward- TTGATCTCCCGGTCAGTTCG } \\
\text { Reverse-CCCATCAGCACAGTTCGTGAG }\end{array}$ \\
GADD45 & NM_001924 & $\begin{array}{l}\text { Forward- AGCGCACGATCACTGTCG } \\
\text { Reverse-CATCTCTGTCGTCGTCCTCGT }\end{array}$ \\
\hline
\end{tabular}

\section{Processing and analysis of biopsies}

Tissue sections ( $3 \mu \mathrm{m}$ thick) were cut from formalin-fixed paraffin-embedded tissue blocks and stained with $\mathrm{H} \& \mathrm{E}$ and immunohistochemical staining using methods described previously. ${ }^{19}$ MIB1 (mindbomb homologue 1 (Drosophila)), a monoclonal antibody against the Ki-67 antigen (1:250 dilution), was used to identify proliferating cells within the tumour tissue. The proliferating cell index (PCI) of the tumours was estimated by examining the stained slides using a square graticule at high magnification $(\times 100)$ as described previously. $^{24} 25$

Biopsies from flat mucosa stored in Carnoy's solution were processed, stained and microdissected as described previously. ${ }^{26}$ Ten intact, non-bifid crypts were counted. The length and breadth of each crypt were measured and the crypt was divided into 10 equal compartments starting from the base to the luminal surface of the crypt. The numbers of mitotic figures in each of the 10 compartments were identified and counted. Crypt microdissection and cell counting were performed by a single histopathologist who was blinded to the patient and treatment details.

A two-step real-time reverse transcriptase-PCR (RT-PCR) using the LightCycler480 SYBR green I Master (Roche Diagnostics, Burgess Hill, Sussex, UK) was used to quantify gene expression in response to starch treatment. RNA was extracted from tissues stored in RNAlater using the SV total RNA isolation system (Promega, Southampton, UK) as described by the manufacturer. Gene expression, relative to expression of the housekeeping gene 18S, was studied for two genes, CDK4 and GADD45A (table 1). In step 1, the reverse transcription of RNA into cDNA was performed outside the LightCycler system using the Moloney murine leukaemia virus RNase $\mathrm{H}^{+}$reverse transcriptase enzyme (Promega). Standard curves for real-time RT-PCR were obtained using Caco 2 cell cDNA at dilutions ranging from 1:5000 to 1:80 000 for the housekeeping gene (18S) and dilutions from 1:5 to 1:160 for the target genes CDK4 and GADD45A. A melting curve was programmed into the LightCycler 480 protocol to access the specificity of the amplified PCR product and to look for any primer dimers, which have a lower melting point when compared with the target product.

RT-PCR amplification of the target genes CDK4 and GADD45 and the housekeeping gene 185 was carried out using the primer oligonucleotides $(0.5 \mu \mathrm{mol})$ detailed in table $1(\mathrm{VH}$ Bio, Gateshead, UK) and the SYBR Green I master kit (Roche). The LightCycler 480 was programmed for one preincubation cycle at $95^{\circ} \mathrm{C}$ for $5 \mathrm{~min}, 45$ mplification cycles at an annealing temperature of $55^{\circ} \mathrm{C}$, one melting curve at $65^{\circ} \mathrm{C}$ and a cooling cycle at $40^{\circ} \mathrm{C}$. The samples were run in duplicate and the amplification curves were compared with the standard amplification curves to obtain the normalised ratio of expression levels of the target genes in the samples. 
Figure 1 Flow chart summarising recruitment and randomisation of patients in Study 1 (adapted from http://www. consort-statement.org/).

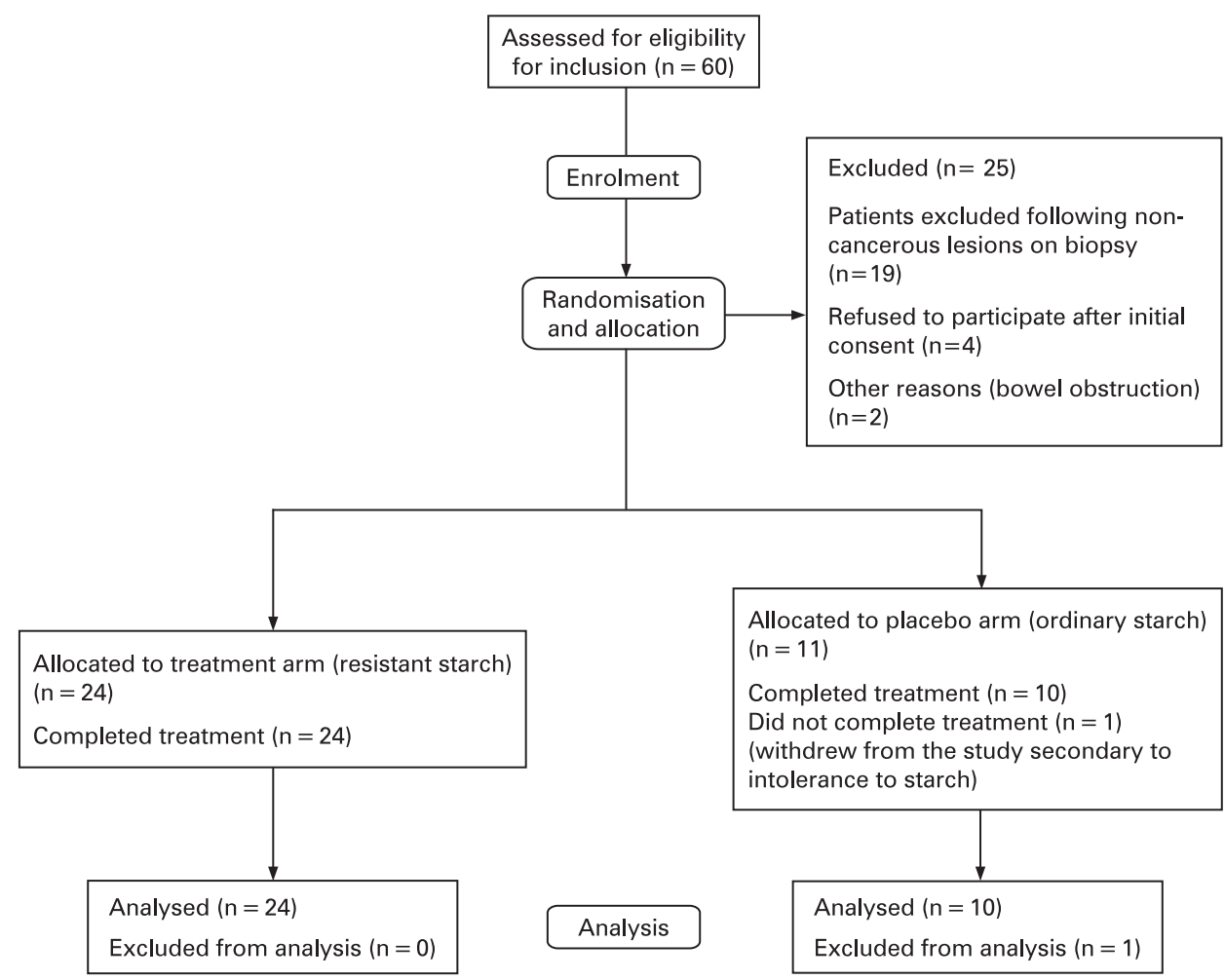

\section{Data collection and statistical methods}

Data were collected in Excel (Microsoft) and Access (Microsoft) databases and analysed using MINITAB version 14 (Minitab, Coventry, UK) and SPSS version 15 (SPSS, Woking, UK) software. The normality of data distribution was tested, and Box Cox transformation was used if the data were not normal. Univariate analysis of variance and covariance were performed using the general linear model. Results are expressed as least square means (SEM), and $\mathrm{p}<0.05$ was considered significant. For Study 1, the effects of the intervention were assessed using analysis of covariance with pretreatment values as a covariate. For Study 2, analysis of variance was used to compare the outcomes in patients receiving treatment starch (RS) as compared with placebo starch. In both studies, the outcomes were adjusted for potential confounding factors including age, sex, site of cancer, preoperative radiotherapy and preoperative immunonutrition by including them as covariates. For posttreatment data only, the results from both studies were pooled and re-analysed with the addition of "study" as a factor in the analysis.

\section{RESULTS}

Of the total 65 patients who were randomised, 62 (95.4\%) patients (35 male; 27 female) completed the starch treatment, of which 34 patients were in Study 1 and 28 patients in Study 2. The details of randomisation and patient flow through the studies have been illustrated in figs 1 and 2. Two patients who stopped taking starch as they did not like the taste and the third patient who underwent emergency surgery were excluded from the final analysis. The demographics, tumour location, details of preoperative treatment and histology of tumours are summarised in table 2. The subjects randomised to each of the two treatment groups (RS and OS) in both studies (Study 1 and Study 2) were comparable in their age and sex distribution, tumour location, preoperative short course radiotherapy and preoperative immunonutrition. Of the 34 patients in Study 1 $26(76 \%)$ patients took starch treatment for $\geqslant 3$ weeks and 8 (24\%) patients took starch for 2 weeks. In Study 2, 18 (64\%) patients took the starch treatment for $\geqslant 3$ weeks and $10(36 \%)$ patients took it for 2 weeks. Following starch treatment, 7 (23\%) patients, of whom 4 patients were on RS and 3 patients were on OS treatment, complained of minor symptoms including a feeling of bloating, flatulence and bad taste, but there were no major side effects. These patients completed the starch treatment after reducing the dose of starch supplements to one sachet per day.

\section{RS and tumour cell proliferation}

In Study 1, paired samples (pretreatment and post-treatment biopsies from each volunteer) were available from 34 patients, of whom 24 were randomised to treatment with RS and 10 were randomised to $O S$ treatment. Across the groups there were no significant differences in the pretreatment (baseline) PCI of tumours (table 3). Following starch treatment, the covariates, site of cancer and percentage of proliferating cells in the pretreatment samples significantly influenced the PCI of tumours, whereas age and sex of the patients had no effect on these measurements. The reduction in PCI was greater for rightside colon cancers (cancers proximal to the splenic flexure) when compared with left-side colon cancers (cancers at and beyond the splenic flexure), and this reduction was seen in both treatment and placebo groups (fig 3). Overall, there was no effect of RS treatment (when compared with OS treatment) on the PCI of tumours in Study $1(p=0.84)$ (table 4).

In Study 2, post-treatment biopsies from tumours were available for 28 patients, of whom 15 were randomised to OS and 13 to RS treatment. There was no significant effect of RS when compared with OS on tumour PCI after adjusting for age, sex, preoperative radiotherapy and preoperative immunonutrition $(p=0.5)$ (table 5). Following analysis of pooled data from 
Figure 2 Flow chart summarising recruitment and randomisation of patients in Study 2 (adapted from http://www. consort-statement.org/).

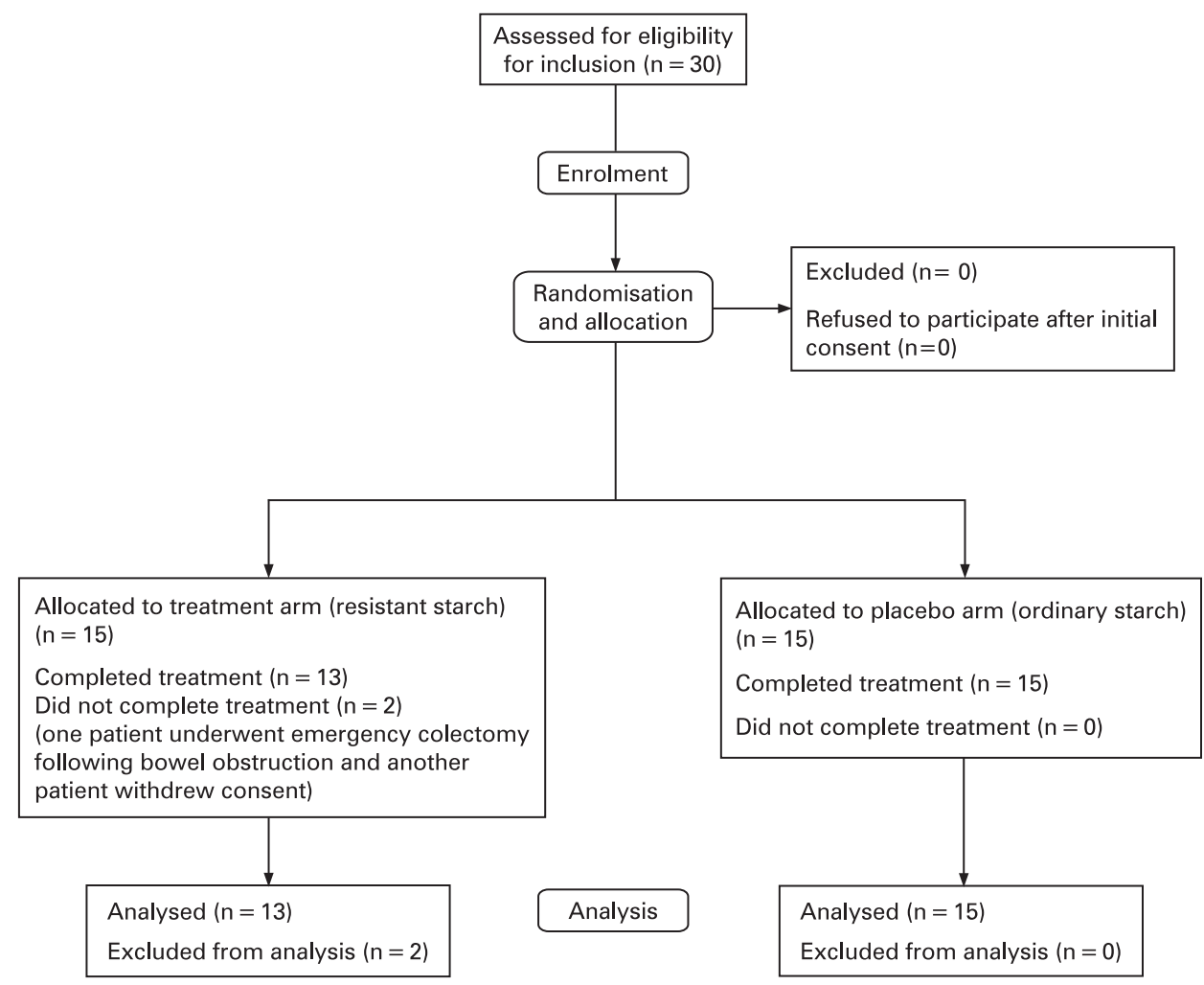

Studies 1 and 2, there was no significant effect of RS on tumour PCI $(p=0.42)$ (table 6).

\section{RS and crypt cell kinetics}

The pretreatment crypt dimensions, mitotic cells per crypt and percentage of mitotic cells in the top half of the crypt for patients in Study 1 have been detailed in table 3. There were more mitotic cells per crypt in the right colon when compared with the left colon $(p<0.01)$, and this intersite difference was seen in both treatment and placebo groups. Following treatment for a median duration of 4 weeks, there were no detectable effects of RS when compared with OS on crypt length, crypt breadth and mean mitotic cell counts per crypt after adjusting for differences in age, sex, site of cancer and pretreatment measurements (table 4).

Table 2 Demographics, tumour location, preoperative treatment and histology of patients in Studies 1 and 2

\begin{tabular}{llll}
\hline & Study 1 & Study 2 & Total \\
\hline No. of patients (male/female) & $34(22 / 12)$ & $28(13 / 15)$ & $62(35 / 27)$ \\
Median age (range), years & $65(35-89)$ & $67(41-78)$ & $66(35-89)$ \\
Family history of CRC & $4(11.8 \%)$ & $6(21.4 \%)$ & $10(16.1 \%)$ \\
Right colon cancer & $9(26.5 \%)$ & $13(46.4 \%)$ & $22(35.5 \%)$ \\
Left colon cancer & $25(73.5 \%)$ & $15(53.6 \%)$ & $40(64.5 \%)$ \\
Preoperative radiotherapy* & $6(17.6 \%)$ & $1(2.9 \%)$ & $7(11.3 \%)$ \\
Preoperative immunotherapy $\dagger$ & $18(52.9 \%)$ & $8(28.6 \%)$ & $26(41.9 \%)$ \\
Histology & & & \\
Moderately differentiated & $32(94 \%)$ & $26(93 \%)$ & $58(93.5 \%)$ \\
Poorly differentiated & $2(5.9 \%)$ & $2(7.1 \%)$ & $4(6.5 \%)$
\end{tabular}

*Preoperative short course radiotherapy consisted of $25 \mathrm{~Gy} / 5$ fractions given over 1 week.

†Preoperative immunonutrition (Oral Impact (Novartis)) consisted of a mixture of omega-3 fatty acids, arginine, antioxidants and soluble fibre given for a week before the planned surgery.

CRC, colorectal cancer.
The proportion of mitotic cells in the top half of the crypt was reduced markedly by RS treatment when compared with placebo, and these differences were statistically significant $(p=0.028)$ after adjusting for differences in age, sex, site of cancer, duration of starch treatment, crypt dimensions and pretreatment measurements (table 4). Further post hoc analysis showed that this reduction in the proportion of mitotic cells in the top half of the crypt following RS treatment was greater in patients aged $\geqslant 75$ years when compared with patients $<75$ years $(p=0.011)$ but, since this was not a predefined analysis, this observation should be treated with caution (fig 4).

In Study 2, RS treatment had no significant effect on crypt length, crypt breadth and total number of mitotic cells within the crypts (table 5). The proportion of mitotic cells in the top half of the crypt was $37 \%$ lower in the RS-compared with the OS-treated subjects, but this difference was not statistically significant $(p=0.14)$ (table 5). When data from Studies 1 and 2

Table 3 Baseline (pretreatment) crypt dimensions and crypt cell kinetics in macroscopically normal mucosa, and proliferation index of tumours for patients in Study 1

\begin{tabular}{llllll}
\hline & $\begin{array}{l}\text { OS } \\
(\mathbf{n}=\mathbf{1 0})\end{array}$ & SEM & $\begin{array}{l}\text { RS } \\
(\mathbf{n}=\mathbf{2 4})\end{array}$ & SEM & p Value \\
\hline Crypt length (nm) & 443 & 34.6 & 470 & 25.8 & 0.55 \\
Crypt width (nm) & 107 & 6.7 & 100 & 5.0 & 0.43 \\
Crypt mitotic cell count & 9.99 & 1.28 & 8.94 & 0.95 & 0.48 \\
$\begin{array}{l}\text { Mitotic cells in top half of crypt } \\
\text { (\%) }\end{array}$ & 13.2 & 3.01 & 8.8 & 2.10 & 0.29 \\
MIB1 proliferation index & 0.47 & 0.04 & 0.45 & 0.03 & 0.71
\end{tabular}

Values in the table are least square means adjusted for age, sex and site of biopsy. Crypt mitotic cell count is the average number of mitotic cells per whole crypt calculated after counting a minimum of 10 microdissected whole crypts from apparently normal flat mucosa. MIB1 proliferation index is the ratio of the number of MIB1-positive cells to total cells in tumour tissue.

MIB1, mindbomb homologue 1; OS, ordinary starch; RS, resistant starch. 
Figure 3 Effects of resistant starch on the proliferating index of tumours in the right and left colon (Study 1). All cancers proximal to the spleenic flexure were grouped as right-side cancers. Values on the $y$-axis are least square means adjusted for differences in age, sex and pretreatment proliferating cell indices. MIB1, mindbomb homologue 1.

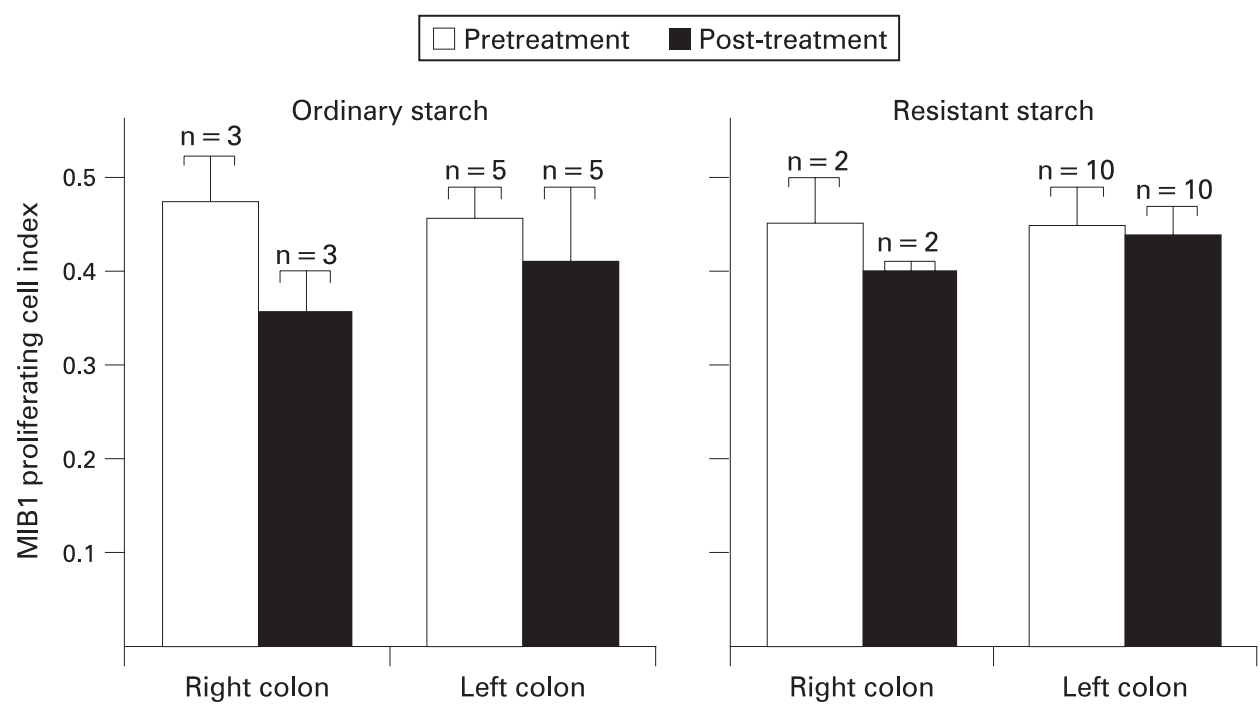

Side of cancer were pooled, the proportion of mitotic cells in the top half of the crypt was 44\% lower in the RS group when compared with the OS group but again the difference was not significant $(p=0.07)($ table 6$)$

\section{RS treatment and gene expression}

In Study 1, paired samples were available for RT-PCR analysis from 26 patients, of whom 9 were randomised to OS treatment and 17 were randomised to RS treatment. Samples from the

Table 4 Crypt dimensions, crypt cell kinetics and tumour proliferation index following starch treatment (Study 1)

\begin{tabular}{llllll}
\hline & $\begin{array}{l}\text { OS } \\
(\mathbf{n = 1 0})\end{array}$ & SEM & $\begin{array}{l}\text { RS } \\
(\mathbf{n}=\mathbf{2 4})\end{array}$ & SEM & p Value \\
\hline Crypt length (nm) & 512 & 27.7 & 520 & 19.3 & 0.82 \\
Crypt width (nm) & 93 & 5.6 & 96 & 3.9 & 0.67 \\
Crypt mitotic cell count & 6.91 & 1.39 & 6.90 & 0.97 & 0.99 \\
$\begin{array}{l}\text { Mitotic cells in top half of crypt } \\
\text { (\%) }\end{array}$ & 13.7 & 3.2 & 3.1 & 1.5 & 0.028 \\
MIB1 proliferation index & 0.41 & 0.02 & 0.42 & 0.02 & 0.84 \\
\hline
\end{tabular}

Values in the table are least square means adjusted for age, sex, site of biopsy and respective pretreatment measurements. Crypt mitotic cell count is the average number of mitotic cells per whole crypt calculated after counting a minimum of 10 microdissected whole crypts from apparently normal flat mucosa. MIB1 proliferation index is the ratio of the number of MIB1-positive cells to total cells in tumour tissue. MIB1, mindbomb homologue 1; 0 S, ordinary starch; RS, resistant starch.

Table 5 Crypt dimensions and crypt cell kinetics in macroscopically normal mucosa and tumour proliferation index following starch treatment (Study 2)

\begin{tabular}{llllll}
\hline & $\begin{array}{l}\text { OS } \\
(\mathbf{n}=\mathbf{1 5})\end{array}$ & SEM & $\begin{array}{l}\text { RS } \\
(\mathbf{n}=\mathbf{1 3})\end{array}$ & SEM & p Value \\
\hline Crypt length (nm) & 432 & 24.8 & 479 & 22.5 & 0.18 \\
Crypt width (nm) & 86 & 2.7 & 84 & 2.5 & 0.58 \\
Crypt mitotic cell count & 6.49 & 0.64 & 6.28 & 0.58 & 0.81 \\
$\begin{array}{l}\text { Mitotic cells in top half of crypt } \\
\text { (\%) }\end{array}$ & 7.3 & 1.3 & 4.6 & 1.1 & 0.14 \\
MIB1 proliferation index & 0.43 & 0.03 & 0.47 & 0.03 & 0.26 \\
\hline
\end{tabular}

Values in the table are least square means adjusted for age, sex and site of biopsy. Crypt mitotic cell count is the average number of mitotic cells per whole crypt calculated after counting a minimum of 10 microdissected whole crypts from apparently normal flat mucosa. MIB1 proliferation index is the ratio of the number of MIB1-positive cells to total cells in tumour tissue.

MIB1, mindbomb homologue 1; OS, ordinary starch; RS, resistant starch.
Table 6 Crypt dimensions and crypt cell kinetics in macroscopically normal mucosa and tumour proliferation index following starch treatment (pooled data from Studies 1 and 2)

\begin{tabular}{llllll}
\hline & $\begin{array}{l}\text { OS } \\
\text { (n= 25) }\end{array}$ & SEM & $\begin{array}{l}\text { RS } \\
(\mathbf{n = 3 7 )}\end{array}$ & SEM & p Value \\
\hline Crypt length (nm) & 461 & 19.5 & 493 & 15.7 & 0.21 \\
Crypt width (nm) & 89 & 2.5 & 89 & 2.0 & 0.96 \\
Crypt mitotic cell count & 6.6 & 0.74 & 6.7 & 0.56 & 0.93 \\
Mitotic cells in top half of crypt & 8.2 & 1.6 & 4.6 & 1.1 & 0.07 \\
(\%) & & & & & \\
MIB1 proliferation index & 0.42 & 0.02 & 0.45 & 0.02 & 0.42
\end{tabular}

Values in the table are least square means adjusted for age, sex, site of biopsy and respective pretreatment measurements. Crypt mitotic cell count is the average number of mitotic cells per whole crypt calculated after counting a minimum of 10 microdissected whole crypts from apparently normal flat mucosa. MIB1 proliferation index is the ratio of the number of MIB1-positive cells to total cells in tumour tissue MIB1, mindbomb homologue 1; OS, ordinary starch; RS, resistant starch.

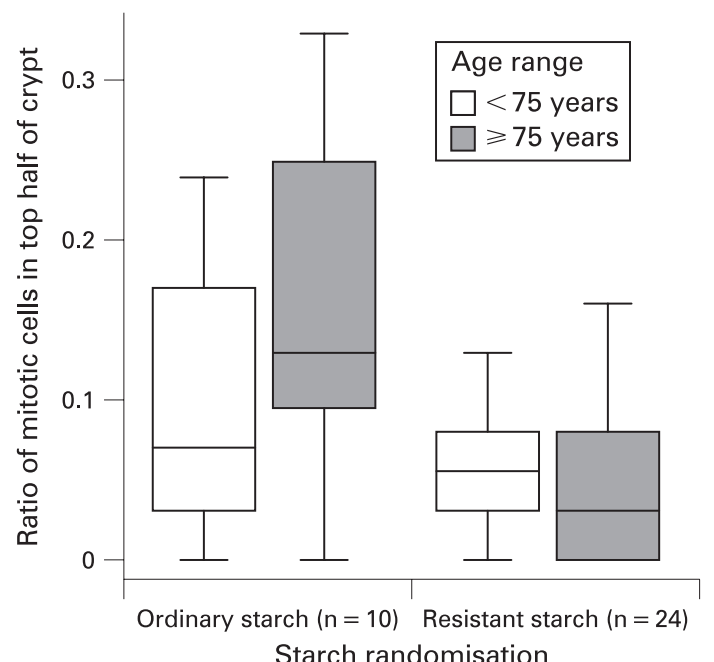

Figure 4 Mitosis in the top half of the colonic crypts following starch treatment in the two age groups (Study 1). Values on the $x$-axis correspond to the number of patients in each treatment group. Values on the $y$-axis are the mean ratio of mitotic cells in the top half of the crypt to the total number of mitotic cells in the crypt (minimum of 10 whole crypts counted for each biopsy). The middle line represents the median, the edges of the boxes represent the 25th and 75th percentiles, and the whiskers represent the maximum and minimum values. 
Table 7 Expression of CDK4 and GADD45A genes in tumour tissue at baseline (Study 1)

\begin{tabular}{llllll}
\hline & OS $(\mathbf{n}=\mathbf{9})$ & SEM & RS $(\mathbf{n}=\mathbf{1 7})$ & SEM & p Value \\
\hline CDK4 & 0.26 & 0.12 & 0.48 & 0.08 & 0.27 \\
GADD45A & 0.53 & 0.12 & 0.40 & 0.08 & 0.23 \\
\hline
\end{tabular}

Values are least square means of the normalised ratios of expression of individual genes compared with expression of the housekeeping gene $18 \mathrm{~S}$ adjusted for age, sex and site of cancer.

$\mathrm{OS}$, ordinary starch; RS, resistant starch.

Table 8 Expression of CDK4 and GADD45A genes in tumours following starch treatment (Study 1)

\begin{tabular}{llllll}
\hline & 0S $(\mathbf{n}=\mathbf{9})$ & SEM & RS $(\mathbf{n}=\mathbf{1 7})$ & SEM & p Value \\
\hline CDK4 & 0.22 & 0.32 & 0.96 & 0.21 & 0.076 \\
GADD45A & 0.14 & 0.52 & 1.54 & 0.42 & 0.064
\end{tabular}

Values are least square means of normalised ratios of expression of individual genes compared with expression of the housekeeping gene $18 \mathrm{~S}$ adjusted for age, sex and site of cancer.

OS, ordinary starch; RS, resistant starch.

Table 9 Differential expression of CDK4 and GADD45A genes in macroscopically normal flat mucosa and tumour tissue (Study 2)

\begin{tabular}{llllll}
\hline & $\begin{array}{l}\text { Normal mucosa } \\
(\mathbf{n = 2 8})\end{array}$ & SEM & $\begin{array}{l}\text { Tumour } \\
(\mathbf{n = 2 8})\end{array}$ & SEM & p Value \\
\hline CDK4 & 0.54 & 0.03 & 0.65 & 0.03 & 0.01 \\
GADD45A & 1.18 & 0.06 & 0.79 & 0.06 & $<0.001$ \\
\hline
\end{tabular}

Values are least square means of normalised ratios of expression of individual genes compared with expression of the housekeeping gene $18 \mathrm{~S}$ adjusted for age, sex and site of cancer.

OS, ordinary starch; RS, resistant starch.

remaining patients in the study could not be used for RT-PCR analysis due to either inadequate quantity of tissue or poor quality of the extracted RNA. Overall there were no differences in the pretreatment expression of CDK4 and GADD45A genes in the tumours from the two treatment groups (table 7). Following RS treatment for a median duration of 4 weeks, expression of both CDK4 and GADD45A genes was markedly upregulated, but these effects failed to achieve statistical significance after adjusting for the differences in age, sex, site of cancer, preoperative radiotherapy and preoperative immunonutrition ( $p=0.076$ and 0.064 for CDK4 and GADD45A genes, respectively) (table 8).

In Study 2, poststarch treatment biopsy samples from tumour and from apparently normal flat colonic mucosa were available for RT-PCR analysis from 15 patients randomised to OS and 13 patients randomised to RS. There was significant upregulation in expression of CDK4 $(\mathrm{p}<0.01)$ and downregulation in expression of GADD $45 A(p<0.001)$ in the tumour tissue when compared with macroscopically normal mucosa (data pooled across both RS and OS treatment groups) (table 9).
Table 11 Expression of CDK4 and GADD45A genes in tumour tissue following starch treatment (data pooled from Studies 1 and 2)

\begin{tabular}{llllll}
\hline & OS $(\mathbf{n}=\mathbf{2 4})$ & SEM & RS $(\mathbf{n}=\mathbf{3 0})$ & SEM & $\mathbf{p}$ Value \\
\hline CDK4 & 0.37 & 0.16 & 0.88 & 0.15 & 0.02 \\
GADD45A & 0.56 & 0.30 & 1.41 & 0.26 & 0.04 \\
\hline
\end{tabular}

Values are least square means of normalised ratios of expression of individual genes compared with expression of the housekeeping gene $18 \mathrm{~S}$ adjusted for age, sex, site of cancer, duration of starch treatment, preoperative immunotherapy, preoperative radiotherapy and pretreatment expression of individual genes.

However, there were no significant differences in the expression of either of these genes following RS treatment when compared with OS treatment both in the tumour tissue and in the flat mucosa (table 10). Analysis of pooled data from both Studies 1 and 2 showed a more than twofold upregulation in expression of both CDK4 and GADD45A genes in tumour tissue in the RS treatment group when compared with the placebo group, and these effects were statistically significant $(p=0.02$ and 0.04 for CDK4 and GADD45A genes, respectively) (table 11).

\section{DISCUSSION}

\section{RS treatment and colorectal cancer cell kinetics}

This is the first randomised human intervention study to investigate the antineoplastic effects of RS in patients with CRC. In animals, RS was shown to decrease mucosal cell proliferation, increase apoptosis and induce changes in gene expression. ${ }^{27}{ }^{28}$ Few studies have attempted to replicate these effects using RS in humans (table 12). Although most of these studies reported an increase in the concentrations of SCFAs and, particularly, butyrate in the colonic lumen, only one study ${ }^{3}$ reported that RS supplementation produced a significant (but small) decrease in total crypt cell proliferation. This has not been confirmed in other studies, including the present study. Alteration in the distribution of mitotic cells in the crypts in normal flat mucosa is one of the earliest detectable premalignant changes occurring in the colon and is observed in the macroscopically normal appearing flat colonic mucosa in patients with CRC. ${ }^{26}{ }^{29-31}$ In the present study, the proportion of mitotic cells in the top half of the crypt was reduced markedly $(p=0.028)$ following RS treatment, and adds support to the hypothesis that RS has antineoplastic effects in the human large bowel. ${ }^{32}$

In tumours, the proportion of mitotic cells, usually expressed as the PCI, is a major determinant of the biological behaviour of cancers, including tumour doubling time, metastatic potential and the response to chemotherapy and radiotherapy. ${ }^{33}$ We detected no effect of RS treatment when compared with OS treatment on tumour PCI, and studies of longer duration and/or larger scale may be necessary to detect such an effect.

It should not be assumed that the observed differences in crypt cell kinetics and PCI of tumours before and after treatment are due entirely to the starch intervention. The

Table 10 Expression of CDK4 and GADD45A genes following starch treatment in tumour and macroscopically normal mucosa (Study 2)

\begin{tabular}{|c|c|c|c|c|c|c|c|c|c|c|}
\hline & \multicolumn{5}{|c|}{ Tumour } & \multicolumn{5}{|c|}{ Normal mucosa } \\
\hline & OS & SEM $(n=15)$ & RS & $\begin{array}{l}\text { SEM } \\
(n=13)\end{array}$ & p Value & OS & SEM $(n=15)$ & RS & $\begin{array}{l}\text { SEM } \\
(n=13)\end{array}$ & p Value \\
\hline CDK4 & 0.44 & 0.07 & 0.49 & 0.07 & 0.67 & 0.70 & 0.28 & 0.30 & 0.26 & 0.32 \\
\hline
\end{tabular}

Values are least square means of normalised ratios of expression of individual genes compared with expression of the housekeeping gene $18 S$ adjusted for age, sex and site of cancer.

OS, ordinary starch; RS, resistant starch. 
Table 12 Summary of human studies investigating antineoplastic effects of resistant starch

\begin{tabular}{|c|c|c|c|}
\hline Author & Study subjects & Type, dose and duration of RS treatment & Effect of RS \\
\hline Van Muster et al ${ }^{3}$ & 14 healthy subjects & $45 \mathrm{~g}$ Hylon VII $(32 \%)$ or $20 \mathrm{~g}$ RS low natural fibre & $\begin{array}{l}\downarrow \text { Cell proliferation, } \uparrow \text { SCFA and butyrate, } \downarrow \text { bile } \\
\text { acids }\end{array}$ \\
\hline Grubben et $a l^{15}$ & $\begin{array}{l}23 \text { patients with recently removed colonic } \\
\text { adenomas }\end{array}$ & $\begin{array}{l}45 \mathrm{~g} \text { amylomaize }(28 \mathrm{~g} \mathrm{RS}) / \text { day or } 45 \mathrm{~g} \\
\text { maltodextrin }\end{array}$ & Cell proliferation NSD, $\uparrow$ butyrate \\
\hline Van Gorkom et $a l^{16}$ & 111 sporadic adenoma patients & $30 \mathrm{~g}$ Hylon VII (19 g RS) or $30 \mathrm{~g}$ of placebo starch & Cell proliferation NSD \\
\hline Wacker et $a l^{17}$ & 12 healthy subjects & Hylon VII (50.7-59.7 g/day) or low RS corn starch & Cell proliferation NSD, $\uparrow$ DNA adducts, $\uparrow$ SCFA \\
\hline
\end{tabular}

NSD, non-significant difference; RS, resistant starch; SCFA, short chain fatty acid.

$\uparrow$ indicates an increase; $\downarrow$ indicates a decrease.

pretreatment samples were collected during colonoscopy while the post-treatment samples were obtained from the resected surgical specimens. Some of the study subjects had received preoperative immunonutrition. Although preoperative immunonutrition may reduce postoperative complication rates and length of hospital stay, ${ }^{34}$ there is no evidence for an effect of immunonutrition on tumour PCI. While all patients undergoing colonoscopy received bowel preparation, most patients having surgery for right-side cancers did not receive bowel preparation. Bowel preparation can affect the colonic microflora, which in turn has been shown to cause an increase in the metaphase index of aberrant crypt foci in normal colonic mucosa, ${ }^{35}$ but the effects (if any) of bowel preparation on tumour PCI are not known.

\section{RS treatment and gene expression}

Alterations in gene expression patterns have been reported in both colon cancer cells and the normal appearing colon mucosa of human colon cancer patients when compared with colonic mucosa from disease-free individuals. ${ }^{36-38}$ In the present study, we investigated the effects of oral supplementation with RS on the expression of two key butyrate-responsive, cell cycle regulatory genes, CDK4 and GADD45A, in CRC tissue and in the normal flat colonic mucosa.

$C D K 4$ is a key cell cycle regulator gene which is overexpressed in a variety of human cancers, ${ }^{39}$ whilst reduced expression of GADD $45 A$ is associated with genomic instability and impaired growth control, and promotes tumourigenesis Results from the present study are consistent with this, showing that the baseline expression of CDK4 was significantly higher whereas the expression of GADD45A was significantly lower in tumour tissue compared with normal flat mucosa (table 9). Analysis of pooled data from Studies 1 and 2 revealed that CDK4 expression in tumours following treatment with RS for a median duration of 4 weeks was twofold higher than that in the OS group $(p=0.02)$ (table 11). It has been shown that butyrate can reduce tumour cell proliferation by inducing cyclin-dependent kinase inhibitors (CKIs), leading to $\mathrm{G}_{1}$ phase cell cycle arrest. ${ }^{40}$ Furthermore, these RS-treated CDK-enriched tumour cells may be targets for CKIs, leading to cell cycle arrest and reduction in cell proliferation.

Similarly, pooled data from both studies showed that GADD45A expression in tumour tissue was more than twofold higher following RS treatment as compared with OS treatment $(p=0.04)$ (table 11). In colorectal cancer cells in vitro, butyrate treatment results in GADD $45 \mathrm{~A}$ induction ${ }^{41}$ and GADD proteins are shown to play a vital role in maintaining genomic stability. ${ }^{42}$ The significant upregulation of its expression in tumour tissue following RS treatment in the present study suggests that GADD45A induction may play an important role in the molecular mechanisms underlying the antineoplastic effects of RS. In a recent study, GADD $45 A$ was one of 12 genes which were downregulated in primary colorectal tumours when compared with adjacent normal mucosa in patients with hepatic metastasis. ${ }^{43}$ Similarly, GADD $45 A$ was downregulated significantly in colonic tumours resistant to 5-flurouracil treatment. ${ }^{44}$ The role of $\mathrm{RS}$ in these patient subgroups needs to be investigated.

\section{CONCLUSIONS}

In summary, this study showed that RS supplementation for up to 4 weeks in patients with CRC reduced the proportion of mitotic cells in the top half of the crypt in macroscopically normal mucosa. Since cell proliferation in the upper part of the crypt is considered as a premalignant marker, its reduction by RS is consistent with an antineoplastic action of this food component. Furthermore, this study showed that RS treatment significantly induced expression of the key cell cycle regulatory genes CDK4 and GADD45A. These changes in gene expression may contribute to the molecular mechanisms underlying the antineoplastic effects of RS.

Acknowledgements: The authors thank Gail Barker and the staff of The CAPP2 Study for their help in providing starch samples for this study, Julie Coaker, Human Nutrition Research Centre, Newcastle University for her help with immuohistochemistry and crypt microdissection, and Allison Howard, Institute of Cell and Molecular Biology, Newcastle University for her help and advice in performing real-time RT-PCR. This study was undertaken as a part of the MD thesis of SSD who conducted the clinical study and wrote the first draft of this article. SSD will be acting as the guarantor of this submission. JMC contributed to real-time RT-PCR assays. SBK cosupervised the project and JB provided support, advice and starch samples. JCM was the principal investigator for the study and was responsible for study design. All authors have contributed towards drafting the manuscript and have approved the final draft submitted.

Funding: This project was funded by the Biotechnology and Biological Sciences Research Council (BBRSC), UK (grant no. 13D/20173).

\section{Competing interests: None.}

Ethics approval: The study protocol and the subsequent amendments were approved by the local regional ethics committees and the research and development department of the local NHS Trust.

Patient consent: All patients enrolled in the study gave a written informed consent for their participation in the study and for the biopsies to be used exclusively for this study. No individual patient-identifiable information was used and all the patient data collected for this study were anonymised.

\section{REFERENCES}

1. Englyst HN, Kingman SM, Hudson GJ, et al. Measurement of resistant starch in vitro and in vivo. Br J Nutr 1996;75:749-55.

2. Muir JG, Yeow EG, Keogh J, et al. Combining wheat bran with resistant starch has more beneficial effects on fecal indexes than does wheat bran alone. Am J Clin Nutr 2004; 79:1020-8.

3. van Munster IP, Tangerman A, Nagengast FM. Effect of resistant starch on colonic fermentation, bile acid metabolism, and mucosal proliferation. Dig Dis Sci 1994;39:834-42.

4. Williams EA, Coxhead JM, Mathers JC. Anti-cancer effects of butyrate: use of micro-array technology to investigate mechanisms. Pro Nutr Soc 2003;62:107-15.

5. Whitehead RH, Young GP, Hayward IP. Effects of short-chain fatty acids on a new human colon carcinoma cell line (LIM1215). Gut 1986;27:1457-63. 
6. Siavoshian S, Blottier HM, Foll EL, et al. Comparison of the Effect of different short chain fatty acids on the growth and differentiation of human colonic carninoma cell lines in vitro. Cell Biol Int 1997;21:281-7.

7. Gamet L, Daviaud D, Denis-Pouxviel C, et al. Effects of short chain fatty acids on growth and differentiation of the human colon-cancer cell line. Int J Cancer 1992;52:286-9.

8. Basson MD, Turowski GA, Rashid Z, et al. Regulation of human colonic cell line proliferation and phenotype by sodium butyrate. Dig Dis Sci 1996;41:1986-93.

9. McIntyre A, Gibson PR, Young GP. Butyrate production from dietary fibre and protection against large bowel cancer in a rat model. Gut 1993;34:386-91.

10. D'Argenio G, Cosenza V, Cave MD, et al. Butyrate enemas in experimental colitis and protection against large bowel cancer in a rat model. Gastroenterology 1996;110:1727-34

11. Thorup I, Meyer 0, Kristiansen E. Effect of potato starch, cornstarch and sucrose on aberrant crypt foci in rats exposed to azoxymethane. Anticancer Res 1995; 15:2101-5.

12. Le Leu RK, Brown IL, Hu Y, et al. Suppression of azoxymethane-induced colon cancer development in rats by dietary resistant starch. Cancer Biol Ther 2007;6:1621-6.

13. Le Leu RK, Brown IL, Hu Y, et al. Effect of dietary resistant starch and protein on colonic fermentation and intestinal tumourigenesis in rats. Carcinogenesis 2007;28:240-5.

14. Toden S, Bird AR, Topping DL, et al. High red meat diets induce greater numbers of colonic DNA double-strand breaks than white meat in rats: attenuation by highamylose maize starch. Carcinogenesis 2007;28:2355-62.

15. Grubben MJ, van den Braak CC, Essenberg M, et al. Effect of resistant starch on potential biomarkers for colonic cancer risk in patients with colonic adenomas: a controlled trial. Dig Dis Sci 2001:46:750-6.

16. van Gorkom BA, Karrenbeld A, van der Sluis $T$, et al. Calcium or resistant starch does not affect colonic epithelial cell proliferation throughout the colon in adenoma patients: a randomized controlled trial. Nutr Cancer 2002:43:31-8.

17. Wacker $\mathbf{M}$, Wanek $P$, Eder $E$, et al. Effect of enzyme-resistant starch on formation of 1,N(2)-propanodeoxyguanosine adducts of trans-4-hydroxy-2-nonenal and cell proliferation in the colonic mucosa of healthy volunteers. Cancer Epidemiol Biomarkers Prev 2002;11:915-20.

18. Iacomino $\mathbf{G}$, Tecce $\mathrm{M}$, Grimaldi $\mathrm{C}$, et al. Transcriptional response of a human colon adenocarcinoma cell line to sodium butyrate. Biochem Biophys Res Commun 2001;285:1280-9.

19. Tabuchi Y, Arai Y, Kondo T, et al. Identification of genes responsive to sodium butyrate in colonic epithelial cells. Biochem Biophys Res Commun 2002;293:1287-94.

20. Daly K, Shirazi-Beechey SP. Microarray analysis of butyrate regulated genes in colonic epithelial cells. DNA Cell Biol 2006;25:49-62.

21. Coxhead JM, Williams EA, Mathers JC. DNA mismatch repair status may influence anti-neoplastic effects of butyrate. Biochem Soc Trans 2005;33:728-9.

22. Joseph J, Mudduluru G, Antony S, et al. Expression profiling of sodium butyrate $(\mathrm{NaB})$-treated cells: identification of regulation of genes related to cytokine signaling and cancer metastasis by NaB. Oncogene 2004;23:6304-15.

23. Asp N-G, van Amelsvoort JMM, Hautvast JGAJ. Nutritional implications of resistant starch. Nutr Res Rev 1996:9:1-31.

24. Going JJ. Counting cells made easier. Histopathology 2006;49:309-11.

25. Marrelli D, Pinto E, Neri A, et al. Mib-1 proliferation index is an independent predictor of lymph node metastasis in invasive breast cancer: a prospective study on 675 patients. Oncol Rep 2006:15:425-9.
26. Mills SJ, Mathers JC, Chapman PD, et al. Colonic crypt cell proliferation state assessed by whole crypt microdissection in sporadic neoplasia and familial adenomatous polyposis. Gut 2001:48:41-6.

27. Le Leu RK, Brown IL, Hu Y, et al. A synbiotic combination of resistant starch and Bifidobacterium lactis facilitates apoptotic deletion of carcinogen-damaged cells in rat colon. J Nutr 2005;135:996-1001.

28. Bauer-Marinovic M, Florian S, Muller-Schmehl K, et al. Dietary resistant starch type 3 prevents tumor induction by 1,2-dimethylhydrazine and alters proliferation, apoptosis and dedifferentiation in rat colon. Carcinogenesis 2006;27:1849-59.

29. Roncucci L, Scalmati A, Ponz de Leon M. Pattern of cell kinetics in colorectal mucosa of patients with different types of adenomatous polyps of the large bowel. Cancer 1991:68:873-8.

30. Anti M, Marra G, Armelao F, et al. Rectal epithelial cell proliferation patterns as predictors of adenomatous colorectal polyp recurrence. Gut 1993;34:525-30.

31. Terpstra OT, van Blankenstein $\mathrm{M}$, Dees J, et al. Abnormal pattern of cell proliferation in the entire colonic mucosa of patients with colon adenoma or cancer Gastroenterology 1987:92:704-8.

32. Rozen $\mathbf{P}$, Fireman Z, Fine N, et al. Oral calcium suppresses increased rectal epithelia proliferation of persons at risk of colorectal cancer. Gut 1989;30:650-5.

33. Spyratos F, Ferrero-Pous M, Trassard M, et al. Correlation between MIB-1 and other proliferation markers: clinical implications of the MIB-1 cutoff value. Cancer 2002;94:2151-9.

34. Zheng Y, Li F, Oi B, et al. Application of perioperative immunonutrition for gastrointestinal surgery: a meta-analysis of randomized controlled trials. Asia Pac J Clin Nutr 2007;16(Suppl 1):253-7.

35. Marotta F, Naito Y, Minelli E, et al. Chemopreventive effect of a probiotic preparation on the development of preneoplastic and neoplastic colonic lesions: an experimental study. Hepato-gastroenterology 2003;50:1914-8.

36. Stulik J, Koupilova K, Osterreicher J et al. Protein abundance alterations in matched sets of macroscopically normal colon mucosa and colorectal carcinoma. Electrophoresis 1999;20:3638-46.

37. Friedman DB, Hill S, Keller JW, et al. Proteome analysis of human colon cancer by two-dimensional difference gel electrophoresis and mass spectrometry. Proteomics 2004:4:793-811.

38. Polley AC, Mulholland F, Pin C, et al. Proteomic analysis reveals field-wide changes in protein expression in the morphologically normal mucosa of patients with colorecta neoplasia. Cancer Res 2006:66:6553-62.

39. Shapiro GI. Cyclin-dependent kinase pathways as targets for cancer treatment. J Clin Oncol 2006;24:1770-83.

40. Schwartz B, Avivi-Green C, Polak-Charcon S. Sodium butyrate induces retinoblastoma protein dephosphorylation, p16 expression and growth arrest of colon cancer cells. Mol Cell Biochem 1998;188:21-30.

41. Chen Z, Clark S, Birkeland M, et al. Induction and superinduction of growth arrest and DNA damage gene 45 (GADD45) alpha and beta messenger RNAs by histone deacetylase inhibitors trichostatin A (TSA) and butyrate in SW620 human colon carcinoma cells. Cancer Lett 2002;188:127-40.

42. Hollander $\mathbf{P}$, Castle G, Joynes JO, et al. Helping patients manage intensified insulin regimens. Nursing 1993:23:48-52.

43. Takata 0, Kawamura YJ, Konishi F, et al. cDNA array analysis for prediction of hepatic metastasis of colorectal carcinoma. Surg Today 2006;36:608-14.

44. de Angelis PM, Fjell B, Kravik KL, et al. Molecular characterizations of derivatives of HCT116 colorectal cancer cells that are resistant to the chemotherapeutic agent 5 fluorouracil. Int J Oncol 2004:24:1279-88. 